terizing those continua which have ends about which they are irreducible. One might then consider the possibility of obtaining interesting decompositions of such continua.

Of course, the work of Kuratowski (especially [2]), as well as that of Thomas [7] are most suggestive. A future work dealing with those ideas is planned.

\title{
References
}

[1] R. H. Bing, Snake-like continua, Duke Math. J. 18 (1951), pp. 653-663.

[2] K. Kuratowski, Theorie des continus irréductibles entre deux points, Tund. Math. 3 (1922), pp. 200-231, and 10 (1927), pp. 225-275.

[3] - Topologie II, Warszawa 1950.

[4] H. C. Miller, On unicoherent continua, Trans. Amer. Math. Soc., 69 (1950), pp. 179-194.

[5] R. L. Moore, Foundations of point set theory, Amer. Math. Soc. Colloquium Publications, vol. 13, Revised Edition, New York 1962

[6] R. H. Rosen, On tree-like continua and irreducibility, Duke Math. J. 26 (1959), pp. 113-122.

[7] E. S. Thomas, Jr., Monotone decompositions of irreducible continua, Rozprawy Matematyczne (Dissertationes Mathematicae), vol. 50 (1966), Warszawa.

[8] G. T. Whyburn, Analytic topology, Amer. Math. Soc. Colloquium Publications, vol. 28 (1942), New York.

Resu par la Rédaction le 30. 12. 1969

\section{On criteria of Blumenthal for inner-product spaces}

\section{by}

Joseph E. Valentine (Logan, Utah)

1. Introduction. The problem of characterizing generalized euclidean spaces has been solved by various authors in many different ways. It is the purpose of this paper to solve the problem along the lines exhibited by Blumenthal [2]. At the same time a generalization of his criteria is obtained and a question asked by Freese in [7] is answered.

2. Four point properties. The following six classes of metric quadruples have been introduced by Wilson, Blumenthal and others.

A metric quadruple $p_{1}, p_{2}, p_{3}, p_{4}$ belongs to class:

$C_{0}$ if and only if $p_{1}, p_{2}, p_{3}, p_{4}$ are pairwise distinct;

$O_{1}$ if and only if $p_{1}, p_{2}, p_{3}, p_{4}$ are pairwise distinct and it contains a linear triple;

$O_{2}$ if and only if $p_{1}, p_{2}, p_{3}, p_{4}$ are pairwise distinct, $p_{3}$ is between $p_{2}, p_{4}$ and $p_{2} p_{3}=p_{3} p_{4}$;

$C_{3}$ if and only if $p_{1}, p_{2}, p_{3}, p_{4}$ are pairwise distinct, $p_{2}, p_{3}, p_{4}$ are linear and $p_{1} p_{2}=p_{1} p_{4}$;

$C_{4}$ if and only if $p_{1}, p_{2}, p_{3}, p_{4}$ are pairwise distinct, $p_{3}$ is between $p_{2}, p_{4}$ and $p_{2} p_{3}=p_{3} p_{4}, p_{1} p_{2}=p_{1} p_{4}$;

$C_{5}$ if and only if $p_{1}, p_{2}, p_{3}, p_{4}$ are pairwise distinct, $p_{3}$ is between $p_{2}, p_{4}$ while $p_{2} p_{3}=2 p_{3} p_{4}$ and $p_{1} p_{2}=p_{1} p_{3}$.

Definition. A metric space has the euclidean, euclidean weak, euclidean feeble, euclidean isosceles weak, euclidean isosceles feeble, euclidean external isosceles feeble four-point property provided every quadruple of its points of class $C_{0}, C_{1}, C_{2}, C_{3}, C_{4}, C_{5}$, respectively, is congruently embeddable in euclidean space.

It is known that in a complete, convex, externally convex metric space the euclidean, euclidean weak, euclidean feeble, and the euclidean external isosceles feeble four-point properties are all equivalent. See [9], [1], [2], [7]. Moreover, each of these properties implies the euclidean isosceles weak and the euclidean isosceles feeble four-point properties. 
Clearly the euclidean isosceles weak four-point property implies the euclidean isosceles feeble four-point property. In this paper we will show that the euclidean isosceles feeble four-point property characterizes generalized euclidean space among the class of complete, convex, externally convex metric spaces. It follows then that all six of the four-point properties are equivalent in a complete, convex, externally convex metric space.

3. The euclidean isosceles feeble flour-point property. From [1] it is known that the euclidean weak four-point property characterizes generalized euclidean spaces among the class of complete, convex, externally convex metric spaces. In order to show that the euclidean isosceles feeble fourpoint property effects such a characterization it suffices to show that such a metric space possessing the euclidean isosceles feeble four-point property also possesses the euclidean wealk four-point property. In the discussion that follows $M$ will denote a complete, convex, externally convex metric space that possesses the euclidean isosceles feeble fourpoint property.

Lewris 1. Each two distinct points of $M$ are the endpoints of exactly one metric segment.

Proof. Since $M$ is complete and convex each two distinct points of $M$ are endpoints of at least one segment. Suppose there are points $p, q$ of $M, p \neq q$, such that $p, q$ are endpoints of at least two segments, say $S_{1}(p, q)$ and $S_{2}(p, q)$. Let $r$ be a point of $S_{1}(p, q)-S_{2}(p, q)$. In traversing $S_{1}(p, q)$ from $r$ to $p$ a first point $u$ of $S_{2}(p, q)$ in encountered, while a first point $v$ of $S_{2}(p, q)$ is similarly met in traversing $S_{1}(p, q)$ from $r$ to $q$. Let $m_{1}, m_{2}$ be the midpoints of the subsegments of $S_{1}(p, q)$ and $S_{2}(p, q)$, respectively, that have only the endpoints $u, v$ in common. It follows from the euclidean isosceles feeble four-point property that $u, v, m_{1}, m_{2}$ are congruently embeddable in the euclidean plane. But this is impossible, for then there would be two points of the euclidean plane without a unique midpoint.

LEMrS 2. Metric segments of $M$ admit unique prolongation.

Proof. Since $M$ is externally convex, the segment $S(s, t)$ may be prolonged. If $S(s, t)$ admits two prolongation, let $p, r$ be points of different prolongations such that $s t p$, str hold and $s t=p t=t r$, and let $q$ denote the midpoint of $p$ and $r$. By the euclidean isosceles feeble four-point property, the quadruples $t, p, q, r$ and $s, p, q, r$ are congruently embeddable in the euclidean plane and consequently
(1)

$$
4 s t^{2}=r s^{2}=q s^{2}+q r^{2},
$$$$
s t^{2}=t r^{2}=t q^{2}+q r^{2},
$$

It follows from the triangle inequality that

(3)

$$
q s^{2}-q t^{2} \leqslant 2 q t \cdot s t+s t^{2}
$$
yields

Subtracting equation (2) from equation (1) and making use of (3)

$$
3 s t^{2}=q s^{2}-q t^{2} \leqslant 2 q t \cdot s t+s t^{2}<3 s t^{2} .
$$

This contradiction proves the lemma.

Combining the results of Lemmas 1 and 2 we have the following lemma.

LeMrS 3. Each two distinct points of $M$ are contained in exactly one line.

Lemira 4. If $p$ is a point of $M$ and $L$ is a line of $M$, then $p$ has exactly one foot on $L$.

Proof. It is known that $p$ has at least one foot say $f$ on $L$. Suppose there is another point $f^{\prime}$ that is a foot of $p$ on $L$. If $q$ is the midpoint of $f, f^{\prime}$, then $p, f, q, f^{\prime}$ are congruently embeddable in the euclidean plane. It follows that $p q<p f$ contradicting the fact that $f$ is the foot of $p$ on $I$ unless $f=f^{\prime}=q$, which completes the proof.

LeMTMa 5. If $L$ is a line of $M, p$ is a point of $M$ not on $L, f$ the foot of $p$ on $L$, and if $q, r$ are points of $L$ such that $q$ is between $r$ and $f$ then $p q$ is less than pr.

Proof. Suppose the contrary. If $p x=p r$ for all $x$ in $S(q, r)$, let $z$ denote the midpoint of $q, r$. It follows from the euclidean isosceles feeble four-point property that $p, q, z, r$ and congruently embeddable in the euclidean plane. But then $p z<p r$, which is the desired contradiction.

If $p x>p r$ for some $x$ in $S(q, r)$ then the function $p x$ assumes it maximum at some point $s$ of $S(q, r)$. Let $p r<k<p s$. Then there are first points $u, v$ of $S(s, f)$ and $S(s, r)$, respectively, encountered (beginning at $s$ ) such that $p u=p v=7$. But if $m$ is the midpoint of $u$ and $v$, by the euclidean isosceles feeble four-point property $p, u, m, v$ are congruently embeddable in the euclidean plane and $p m<p u$, contrary to $u, v$ having distance from $p$ no greater than any point of $S(u, v)$.

Lemma 6. If $p$ is a point of $M I$ not on a line $L$ of $M$, and if $q, r$ are points of $L$ with $p q=p r$, then $m$, the midpoint of $q$ and $r$ is the foot of $p$ on $L$.

Proof. By Lemmas 4 and 5 , the foot of $p$ on $L$ lies between $q$ and $r$. If $m$ is not the foot of $p$ on $L$, assume the labeling such that the foot $f$ of $p$ on $L$ is between $q$ and $m$. Since the function $p x$ is continuous and monotone increasing as $x$ recedes along either half-line of $L$ determined by $f$, there is a point $m^{\prime}$ between $q$ and $m$ such that $p m^{\prime}=p m$. If $m^{*}$ is the midpoint of $m$ and $m^{\prime}$, then by the euclidean isosceles feeble four-point 
property the quadruples $p, q, m, r$ and $p, m, m^{*}, m^{\prime}$ are congruently embeddable in the euclidean plane. It follows that

$$
p r^{2}=p m^{* 2}+m^{*} r^{2}-2 m r \cdot m m^{*}
$$

and

$$
p q^{2}=p m^{* 2}+q m^{* 2}+2 q m \cdot m m^{*} .
$$

Subtracting equation (4) from equation (5) and making use of $m * r$ $=m^{*} m+m r$ and $m r=q m=q m^{*}+m m^{*}$ yields $m m^{*}=0$ or $m=m^{*}$. This contradiction completes the proof.

Leimana 7. The space $M$ has the euclidean isosceles weat four-point property.

Proof. Suppose $p, q, r$ are points of $M$ such that $p q=p r$ and suppose $s$ is any point on the line $L(q, r)$ through $q$ and $r$. If $s=f$, the foot of $p$ on $L(q, r)$ the proof is complete by Lemma 6 . Suppose then that $s \neq f$ and let $p^{\prime}, q^{\prime}, r^{\prime}$ be points of the euclidean plane which are congruent to $p, q, r$. It follows by Lemma 6 that $p f=p^{\prime} f^{\prime}$, where $f^{\prime}$ is the foot of $p^{\prime}$ on the line $L\left(q^{\prime}, r^{\prime}\right)$. Let $s^{\prime}$ denote the point corresponding to $s$ under the obvious congruence of $L(q, r)$ with $L\left(q^{\prime}, r^{\prime}\right)$ and let $t$ be the point of $L(q, r), t \neq s$, such that $p s=p t$. If follows from Lemma 6 that $p, t, f, s$ are congruently embeddable in the euclidean plane and consequently $p s^{2}=p f^{2}+f s^{2}=p^{\prime} f^{\prime 2}+f^{\prime} s^{\prime 2}=p^{\prime} s^{\prime 2}$. Hence, the quadruple $p, q, r, s$ is congruent to the quadruple $p^{\prime}, q^{\prime}, r^{\prime}, s^{\prime}$ of the euclidean plane and the proof is complete.

LEMMA 8. The space $M$ has the euclidean weak four-point property.

Proof. Let $p, q, r, s$ be any four points of $M$ such that $q, r, s$ are linear and $p$ is not on the line $L$ that contains them. No generality is lost in assuming $q$ is not the foot of $p$ on $L$. Then there is a point $u$ on $L$ such that $p u=p q$. By Lemma 7 points $p^{\prime}, q^{\prime}, u^{\prime}, r^{\prime}$ and $p^{*}, q^{*}, u^{*}, s^{*}$ of the euclidean plane exist such that $p, q, u, r$ are congruent to $p^{\prime}, q^{\prime}, u^{\prime}, r^{\prime}$ and $p, q, u, s$ are congruent to $p^{*}, q^{*}, u^{*}, s^{*}$. Thus a motion of the euclidean plane exists which sends $p^{*}, q^{*}, u^{*}$ onto $p^{\prime}, q^{\prime}, u^{\prime}$, respectively, and $s^{*}$ onto a point say $s^{\prime}$. Let $f, f^{\prime}$ denote the respective feet of $p, p^{\prime}$ on $L(q, r)$ and $L\left(q^{\prime}, r^{\prime}\right)$. Then by Lemma $6, p f=p^{\prime} f^{\prime}$ and $p^{\prime} s^{\prime 2}=p^{\prime} f^{\prime 2}+f^{\prime} s^{\prime 2}$ $=p f^{2}+f s^{2}=p s^{2}$. Thus the quadruple $p, q, r, s$ is congruently embeddable in the euclidean plane.

The criteria of Blumenthal [1] is now satisfied and we have the following characterization.

THEOREM. A complete, convex, externally convex metric space is generalized euclidean if and only if it has the euclidean isosceles feeble four-point property.

\section{References}

[1] L. M. Blumenthal, Theory and applications of distance geometry, Oxford 1953.

[2] - An extension of a theorem of Jordon and von Neumann, Pacific J. Math. 5 (1955), pp. 161-167.

[3] - and E.Z. Andalafte, Metric characterizations of banach and euclidean spaces, Fund. Math. 55 (1964), pp. 23-55.

[4] M. M. Day, Some characterizations of inner product spaces, Trans. Amer. Math. Soc. 62 (1947), pp. 320-337.

[5] - On criteria of Kasahara and Blumenthal for inner product spaces, Proc. Amer. Math. Soc. 10 (1959), pp. 92-100.

[6] M. Frechet, Sur la definition axiomatique d'une classe d'espaces vectoriels distancies applicables vectorellement sur l'espace de Hilbert, Ann. of Math. 36 (1935), pp. 705-718.

[7] R. W. Freese, Criteria for inner product spaces, Proc. Amer. Math. Soc. 19 (1968), pp. 953-958.

[8] P. Jordon and J. von Neumann, On inner products in linear metric spaces, Ann. of Math. 36 (1935), pp. 719-723.

[9] W.A. Wilson, A relation between metric and euclidean spaces, Amer. J. Math. 54 (1932), pp. 505-517.

UTAF STATE UNIVERSITY

Reçu par la Rédaction le \%. 4. 1970 